\title{
A novel pipelined carry adder design based on half adder
}

\author{
Salah Hasan Alkurwy, Isam Salah Hameed \\ Department of Electronic Engineering, College of Engineering, University of Diyala, Baqubah, Iraq
}

\begin{tabular}{l}
\hline Article Info \\
\hline Article history: \\
Received Jul 19, 2020 \\
Revised Nov 30, 2021 \\
Accepted Dec 10, 2021 \\
\hline Keywords: \\
Carry look-ahead adder \\
Half adder \\
Pipelined carry adder \\
Ripple carry adder
\end{tabular}

\begin{abstract}
A new design of binary parallel adder circuit is presented in this paper. The pipeline technique is applied to implement a group of a half adder (HA) blocks to architect the proposed adder. The pipelined carry adder (PCA) method is suitable for carrying out the desired adder by using the HA circuits of XOR and AND gates. The applied technique reduces the critical path delay by $27 \%$ compared with the ripple carry adder (RCA) and relatively lowers logic gates by 55\% compared with the carry look-ahead adder (CLA). The coded design of the proposed circuit is implemented and simulated on the Cyclone IV FPGA kit platform. Results show that the circuit needs a $7.69 \eta$ Sec delay time to provide the output values. The suggested PCA circuit is more attractive than the conventional ripple carry adder for future electronic applications.
\end{abstract}

This is an open access article under the CC BY-SA license.

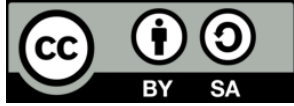

\section{Corresponding Author:}

Salah Hasan Alkurwy

College of Engineering, University of Diyala

32001 Baqubah, Diyala, Iraq

Email: salahalkurwy@uodiyala.edu.iq

\section{INTRODUCTION}

Various electronic devices have been rapidly developed in recent years with the emergence of new methods and techniques to improve the circuits in these devices. An adder is a combinational circuit which is important in improving arithmetic circuits. The structure of the full adder circuit explains the summation of three bits. A group of full adder circuit is serially connected to obtain the computation of the N-bit parallel numbers.

The critical path delay is determined by the longest pathway through the adder circuit. Therefore, the critical path delay of $\mathrm{N}$-bit adders is determined by three gate delays of the first bit adder and to ripples in two gate delays of each of the next adders [1], explaining why it is called 'ripple carry adder (RCA)' [2], [3]. The critical path delay is calculated using (1).

$$
c_{\text {out }}=2 N+1
$$

where $\mathrm{N}$ is the number of the bit adder.

The delay time of parallel adders hinders the use of RCA. Thus, various techniques and methods have been used to minimize the path delay. A commonly used method in addition to the RCA, is the carry look-ahead adder (CLA). This method is based on calculating the result quickly for each stage [4], [5], considering that the carry-in of the previous stage will have 0 or 1 and the critical path delay is achieved only after two gate delays (Figure 1). The fast-calculating feature of the CLA is applied in [6], [7] to use 32-bit and 64-bit CLAs, respectively. The designed CLA circuit in [6] can switch between the approximate and exact modes in error-resilient and execute applications. The disadvantage of the CLA method is the exponentially increasing AND-OR gates with the growing bit number. The critical path delay of the 8-bit 
CLA in (2) declares how the path delay requires multi-bit OR-AND gates to achieve the correct carry-out value.

$$
\begin{aligned}
& C_{\text {out }}=g_{7}+p_{7} g_{6}+p_{7} p_{6} g_{5}+p_{7} p_{6} p_{5} g_{4}+p_{7} p_{6} p_{5} p_{4} g_{3}+p_{7} p_{6} p_{5} p_{4} p_{3} g_{2} \\
& +p_{7} p_{6} p_{5} p_{4} p_{3} p_{2} g_{1}+p_{7} p_{6} p_{5} p_{4} p_{3} p_{2} p_{1} g_{0}+p_{7} p_{6} p_{5} p_{4} p_{3} p_{2} p_{1} p_{0} C_{0}
\end{aligned}
$$

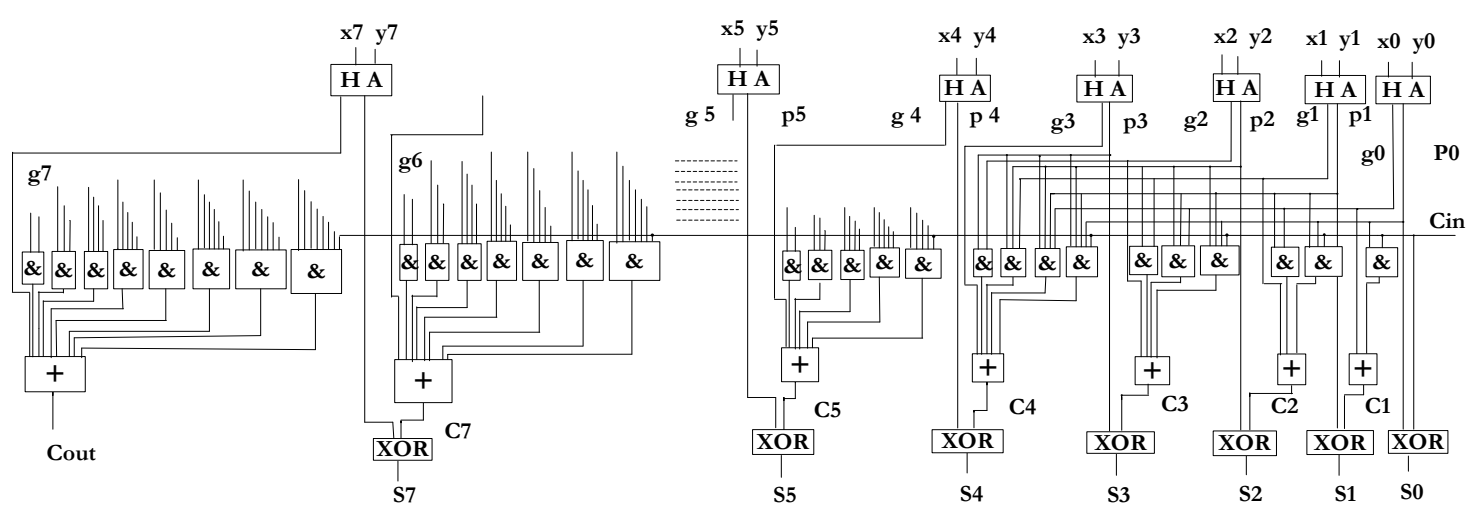

Figure 1. Block circuit diagram of the 8-bit CLA

Another method is used to architect the parallel adder by inserting two rows of RCAs, namely, carry select adder, with carry-in input for 0 and 1 [8], [9]. A $2 \times 1$ multiplexer is used for each bit to provide the sum output, as shown in Figure 2. The critical path output of both RCAs is joined to the multiplexer to select the carry out of the couple RCAs.

Pipelined technique is used to improve the processing of system performance by parallel feeding data inputs to the systems [10]-[13], and efficiently reduced the requested time delay. Deep learning, is a function of artificial intelligence (AI), and it's a part of the machine learning methods that use the stochastic computing to imitate the human brain for processing data. In recent years, this technique is rapidly developed and used for the optimized implementations in various fields such as image classifications [14], computer vision and speech recognition. Deep learning technology also used to optimize the electronic logic circuit using Markov decision process (MDP) [15], and for the analog circuit as in [16].

Deep and machine learning algorithms are a good solution to optimize the power, delay and area efficient of parallel prefix adder design with wide (32-bit, 64-bit) ranges [17], [18] such as Brent-Kung, Kogge-Stone and Sklansky adders. As the proposed sample design is a small bit circuit (8-bit adder), the artificial intelligence methods become not useful to use in this design.

This paper presents a new design of binary parallel adder circuit. The proposed adder is prepared by implementing the pipeline stages of half adder (HA) circuits to architect two samples of 8-bit pipelined parallel adder. Then, the HA circuit of the suggested design is configured based on 3-, and 4-transistors complementary metal-oxide-semiconductor (CMOS) logic gates in order to reduce the increasing of the number of CMOS transistors proportional with growing $\mathrm{N}$-bits adder. The research paper is organized as follows: section 1 provides the introduction. Section 2 describes the design circuit. Section 3 contains the results and discussion. Section 4 ends with a conclusion.

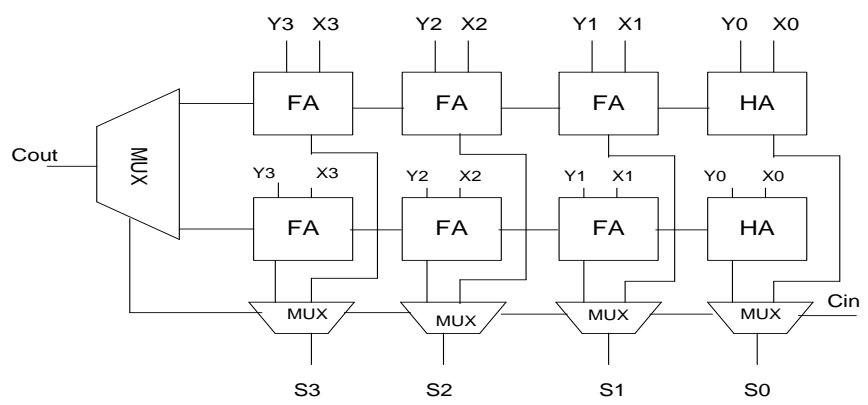

Figure 2. Block circuit diagram of carry select adder 


\section{DESIGN CIRCUIT OF PIPELINED CARRY ADDER}

A novel method of binary parallel adder circuit is designed based on the HA circuits to provide the critical path delay with minimum gate delay. Pipelining connects the HA to the XOR inputs of the other HA blocks in the next level. The proposed pipelined carry adder (PCA) method can enable the circuit to provide the sum outputs of the parallel adder through the stages, whereas the critical path carries out as the group of the all stages in the OR gate. Figure 3 shows that the presented PCA needs only HA circuits to architecture the desired parallel adder. The path delay time is calculated by the number of stages with one OR gate.

It can be formulated by (3).

$$
c_{\text {out }}=N+1
$$

Where $\mathrm{N}$ is the binary digit bits. In (3) shows that the path delay of carry output required N-bit delays, farther one more gate delay time. This describes, why the delay time of the proposed method is about $27 \%$ time less than that in the RCA.

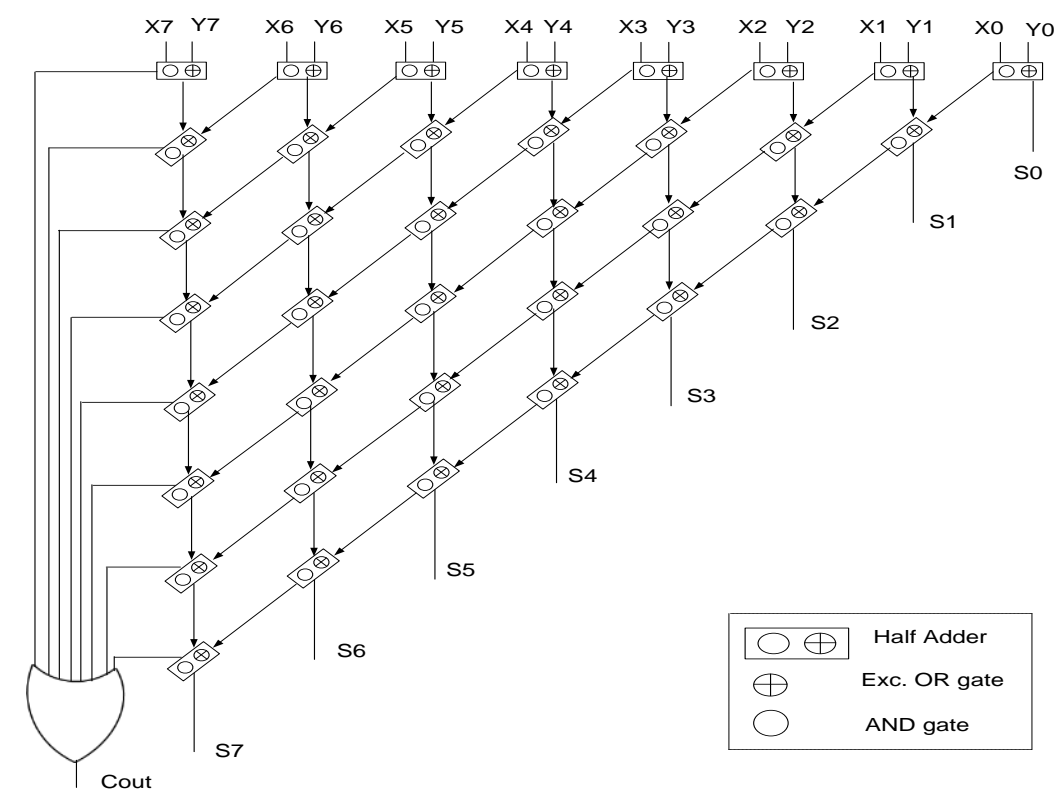

Figure 3. Circuit diagram of the proposed 8-bit pipelined carry adder

\subsection{Modified pipelined carry adder (PCA)}

The proposed PCA design circuit relatively reduces the carry out path-delay compared with the RCA. This advantage is accompanied by increasing the number of HA circuits proportional to increasing the number of bit adders. Calculation of the HA circuits used is based on (4).

$$
\text { No. of HA blocks }=\frac{N}{2}(N+1)
$$

The proposed design is modified by partitioning the given bit numbers into a small circuit of two and three bits (Figure 4) to avoid the cumulative increase in the HA circuit with the wider PCA bit adder while preserving the desired path delay. The proposed modification technique can slightly increase the path delay time, but can greatly reduce the number of HA circuits by up to $45 \%$. As an example, the 8-bit PCA (Figure 3) requests 36 blocks of HA circuits, whereas the modified circuit of the same desired bit adder requires only $20 \mathrm{HA}$ circuits with an additional AND gate to each mini circuit (Figure 4).

As an example of the proposed 8-bit PCA circuit in Figure 4, the summation of two parallel bits of 10010101 and 11010101 is clearly described in Figure 4. The appearing ( 0 and 1$)$ digit numbers in the dashed circles describes the exact operating process. The result will be $101101010(149+213=362$ in decimal). The effect of the suggested new modification technique to the PCA design will clearly demonstrate how this technique can relatively reduce the number of HA circuits, especially with wider (16-bit, 32-bit) parallel adders compared to the RCA or CLA. 

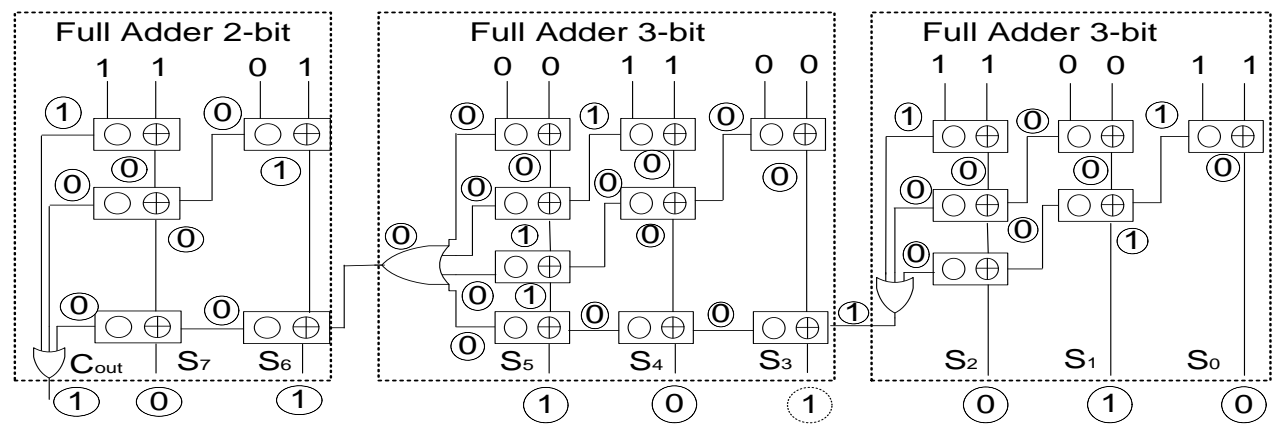

Figure 4. Description of the sum of two parallel bits in PCA

\subsection{Configuration of HA circuit based on CMOS gates}

In recent years, the complementary metal-oxide-semiconductor (CMOS) technology has become the first choice for the fabrication of electronic circuits and devices. CMOS is a metal-oxide-semiconductor field-effect transistor (MOSFET) manufacturing process which uses the n- and p-types in a logic circuit which is complementary connected. The proposed PCA is designed based on HA circuits; therefore, the circuit performance must be improved by minimizing the transistor counts of the XOR CMOS logic.

The basic half adder circuit block consists of the XOR and AND logic gates. The XOR gate was early manufactured in 8-transistors based on CMOS logic [19], 6- transistors [20], [21] and 4-transistors [22], [23]. The power consumption of the circuit decreases when the number of transistors used is reduced. Hence, designers aim to use a small number of transistors to build the XOR gate. Preparing a CMOS 3-transistor XOR gate is a good choice as in [24]-[26]. Given that the proposed 8-bit PCA is designed based on HA, the desired circuit is generated by a mini-number transistor XOR gate. Only two transistors are used in the optimized 2-bit XOR CMOS gate configuration [27]. The circuit diagram of the 2-T XOR gate is shown in Figure 5(a). Using the multiplexer case, the 2-bit AND gate with two transistors [25] can be found as in the Figure 5(b).

The above explanation details enable the construction of the 4-transistor half-adder circuit, as shown in Figure 6(a). The HA circuit is also constructed as a managed circuit of the XOR and AND gates based on CMOS logic. The provided circuit decreases the power consumption of HA. Only three transistors are needed to obtain the desired HA circuit [28], as shown in Figure 6(b).

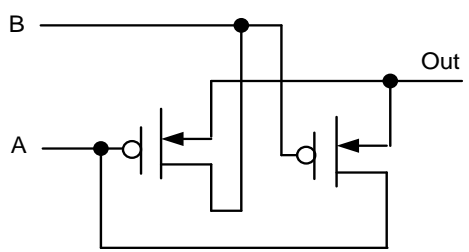

(a)

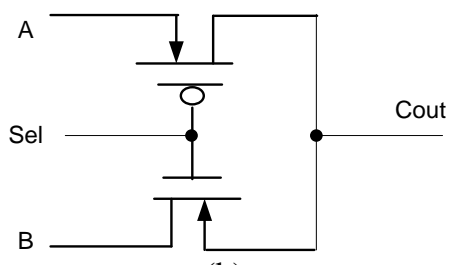

(b)

Figure 5. 2- transistors circuit diagram of (a) XOR gate and (b) MUX AND gate

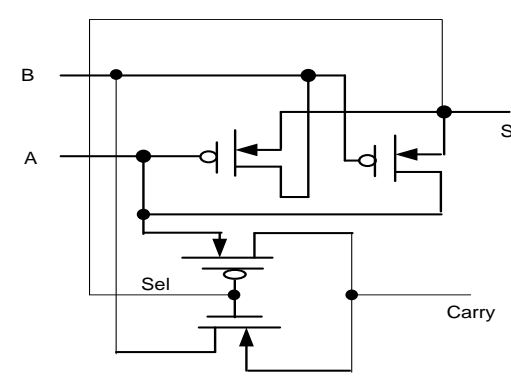

(a)

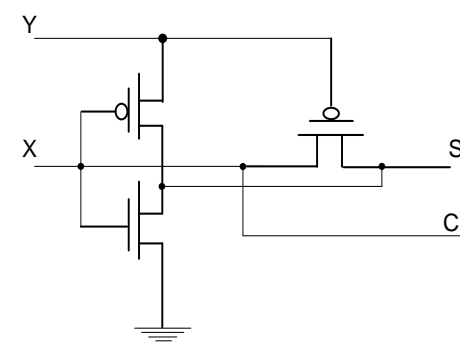

(b)

Figure 6. Half adder circuit diagram-based No. of transistors: (a) 4-transistor and (b) 3- transistors 
For the proposed 8-bit PCA to use the suggested 3-transistor HA, the circuit must be partitioned into three parts based on the diagram circuit in Figure 4. The proposed model full circuit is shown in Figure 7. The figure defines that the CMOS logic includes $86 \mathrm{n}$ - and p-type MOSFET transistors. This is the result of the 20 of 3-transistor HAs, two 3-bit and one 4-bit OR gates.

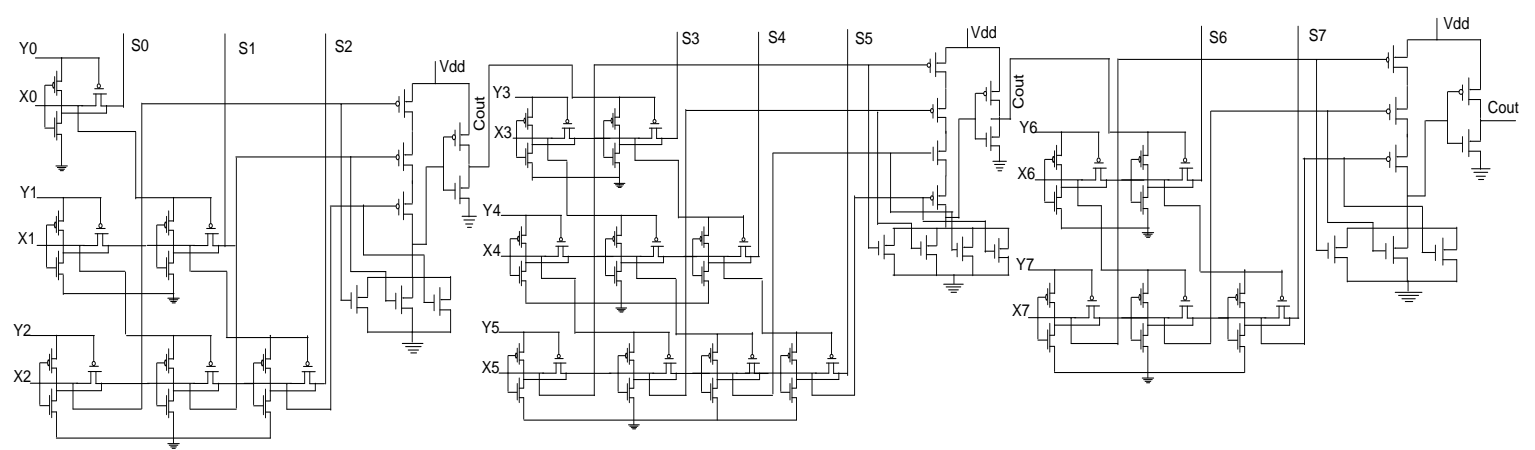

Figure 7. 8-bit PCA circuit diagram-based CMOS logic

\section{RESULTS AND DISCUSSION}

The calculation of two binary values provides a result $(00,01,10$ or 11$)$. The HA is a suitable circuit to obtain the given result. For the summation of multi binary digit bits, then the circuit diagram of the parallel adders is complicated to overcome the carried out value of each two binary bits, which is the critical pathway. The designers applied multiple methods and various techniques to reduce the critical path delay and therefore enhance the circuit's performance while preserving correct results. As shown in Figure 7, the block circuit diagram of the proposed PCA design was structured with the three OR gates based on the groups of 20 HA (3-transistor) circuit.

In addition to the number of count transistors, a comparison of the proposed PCA and the other parallel adders in terms of gate delay and logic gates is given in Table 1 . Table 1 shows the advantage of the proposed design compared to the RCA in terms of gate-delay units. In specific, a gate delay reduction of $27 \%$ can be achieved by the PCA and a logic gate reduction of 55\% can be achieved by the CLA. The CLA has a low gate delay time, but its number of logic gates exponentially increases with increasing wide bit numbers. The number of transistors of the proposed design circuit compared with the different techniques described in Table 1 shows clearly lower transistor counts. The 3-transistor circuit could be used to architect the proposed design, which could reduce the transistors amount and power usage of the circuit.

Table 1. Comparison of the proposed PCA with other reported works

\begin{tabular}{ccccc}
\hline Parameter 8-bit & Gate delay & Logic gates & Technique & No of Transistors \\
\hline RCA & 15 & 37 & CMOS & $128[29]$ \\
& & & & $224[30]$ \\
CLA & 3 & 68 & TG logic & $176[31]$ \\
& & & CMOS & $408[32]$ \\
CSA & 15 & 52 & m-GDI & $110[32]$ \\
This work & 11 & 43 & 3-Tatic CMOS & $354[33]$ \\
& & & 4-Trans. CMOS & 86 \\
& & & & \\
\hline
\end{tabular}

The modified PCA design circuit has been coded, synthesized and elaborated in Quartus II software. The register transfer level viewer of the proposed design circuit and the portioned 3-bit adder is shown in Figures 8(a) and (b). The coded design circuit of the modified 8-bit PCA was implemented on the Cyclone IV-GX FPGA kit platform and simulated using ModelSim software to verify the functionality of the proposed design. The gate level simulation result is shown in Figure 9. 


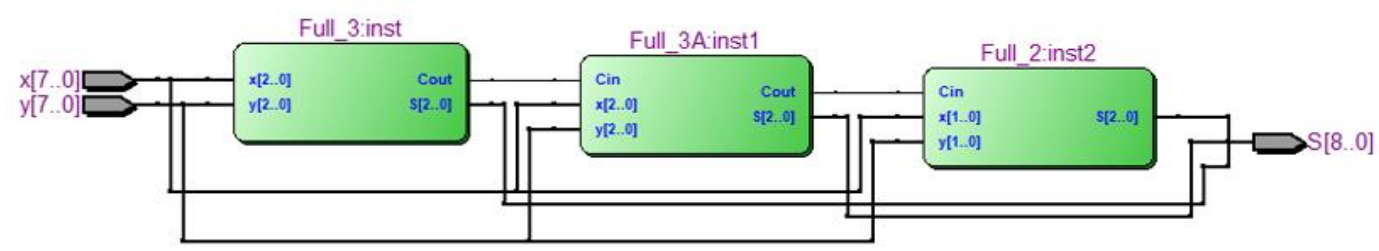

(a)

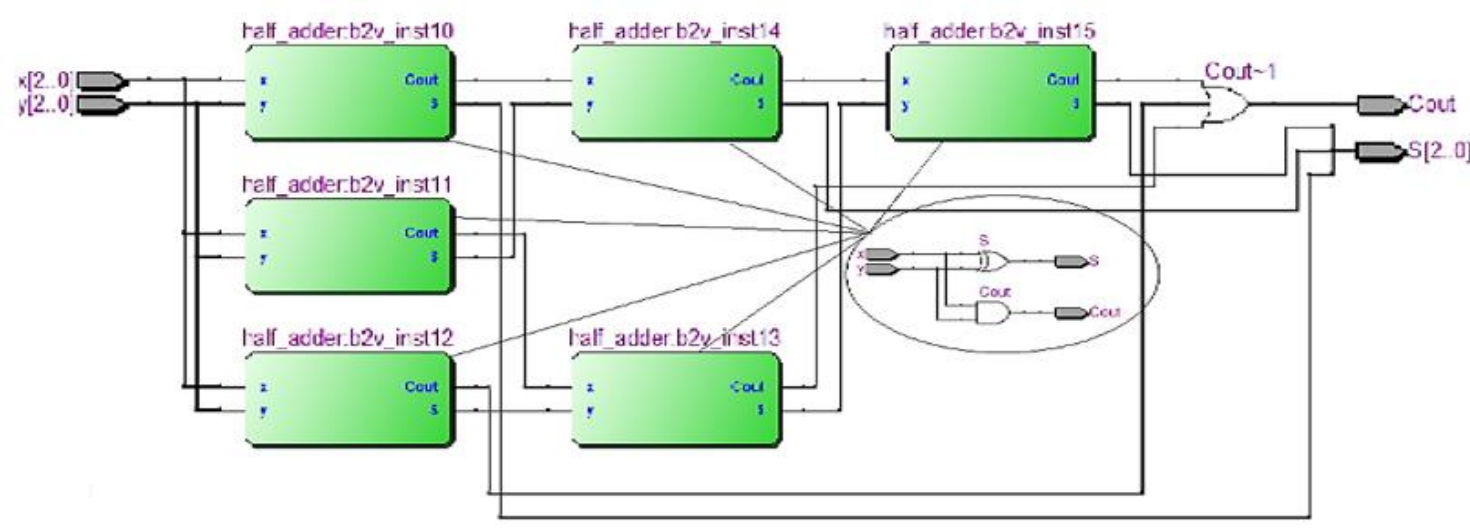

(b)

Figure 8. The created register transfer level viewer for: (a) 8-bit PCA and (b) portioned 3-bit adder

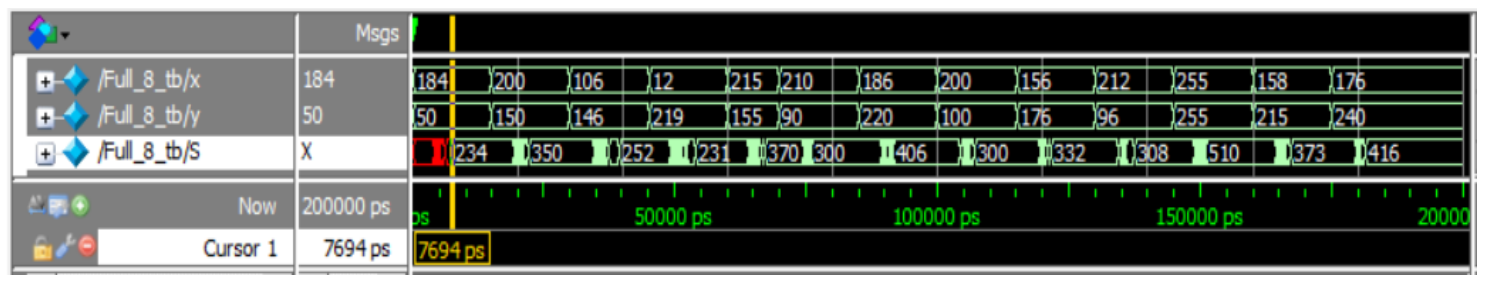

Figure 9. Gate level simulation of the 8-bit PCA adder

\section{CONCLUSION}

A novel method of the PCA circuit is designed and implemented in this paper. The proposed design circuit is an architect based on the HA circuits. The 8-bit PCA is proposed in two design circuits. The pipelined PCA circuit is architected based on the HA blocks to provide the correct sum with a short path delay. The second modified PCA is established by partitioning the 8-bit into small circuits of two-bit and three-bit adders to reduce the HA block circuits. The modified PCA circuit was coded Verilog HDL code, elaborated in Quartus II software and then implemented and simulated on the Cyclone IV-GX FPGA platform software, to verify the functionality results of the circuit. The proposed PCA is easy to design because of the use of the HA circuit with a lower number of transistors compared with the other works. The design relatively reduces the critical path delay by $27 \%$ of the PCA and lower logic gate by $55 \%$ of the CLA.

\section{REFERENCES}

[1] B. Koyada, N. Meghana, Md. O. Jaleel, and P. R. Jeripotula, "A comparative study on adders," in International Conference on Wireless Communications, Signal Processing and Networking (WiSPNET), Chennai, India, 2017, pp. 2226-2230, doi: 10.1109/WiSPNET.2017.8300155.

[2] D. Rajkumar, P. K. Dutta, and S. K. Sarkar, "Design and implementation of 4-bit ripple carry adder using SETMOS architecture," in 2016 IEEE International Conference on Recent Trends in Electronics, Information \& Communication Technology (RTEICT), Bangalore, India, 2016, pp. 58-61, doi: 10.1109/RTEICT.2016.7807782.

[3] M. Sarkar, G. S. Taki, Prerna, and S. N. Ray, "Design of ripple carry adder using CMOS output wired logic based majority gate," in 8th Annual Industrial Automation and Electromechanical Engineering Conference (IEMECON), Bangkok, Thailand, 2017, pp. 228- 231, doi: 10.1109/IEMECON.2017.8079617.

[4] S. Brown and Z. Vranesic, Fandemantals of Digital Logic With Verilog Design. Singapore: McRaw-Hill, 2014, pp. 146-150. 
[5] S. Alkurwy, S. Ali, S. Islam, and Faizul Idros, "A low power memoryless ROM design architecture for a direct digital frequency synthesizer," Turkish Journal of Electrical Engineering \& Computer Sciences, vol. 25, no. 5, pp. 4023-4032, 2017, doi: 10.3906/elk-1609-61.

[6] O. Akbari, M. Kamal, A. A. Kusha, and M. Pedram, "RAP-CLA: A Reconfigurable Approximate Carry Look-Ahead Adder," in IEEE Transactions on Circuits and Systems II: Express Briefs, vol. 65, no. 8, pp. 1089-1093, Aug. 2018, doi: 10.1109/TCSII.2016.2633307.

[7] R. Bank and S. Mangaraj, "Design and Implementation of 64-bit Carry Lookahead Adders Using Fixed and Variable Stage Structure," in 4th International Conference on Devices, Circuits and Systems (ICDCS), Coimbatore, India, 2018, pp. 143-147, doi: 10.1109/ICDCSyst.2018.8605162.

[8] B. Amelifard, F. Falah, and M. Pedram, "Closing the gap between carry select adder and ripple carry adder: a new class of low-power high-performance adders," Sixth international symposium on quality electronic design (isqed'05), San Jose, CA, USA, 2005, pp. 148-153, doi: 10.1109/ISQED.2005.131.

[9] M. Soundharya, P. G. Scholar, and R. Arunkumar, "GDI based area delay power efficient carry select adder," in International Conference on Green Engineering and Technologies (IC-GET), Coimbatore, India, 2015, pp 1-5, doi: 10.1109/GET.2015.7453845.

[10] S. Alkurwy, S. H. Ali, Md. S. Islam, and F. Idros, "An area efficient memory-less ROM design architecture for direct digital frequency synthesizer," International Journal of Electrical and Computer Engineering (IJECE) vol. 11, no. 1, pp. 257-264, 2021, doi: 10.11591/ijece.v11i1.pp257-264.

[11] A. Khalaf, M. Nabil, and S. M. Hassan, "Design and implementation of pipelined and parallel AES encryption systems using FPGA," Indonesian Journal of Electrical Engineering and Computer Science, vol. 20, no. 1, pp. 287-299, October 2020, doi: 10.11591/ijeecs.v20.i1.pp287-299.

[12] S. H. Ibrahim, S. H. M. Ali, and M. S. Islam, "Design a 24-bits pipeline phase accumulator for direct digital frequency synthesizer," 2012 International Symposium on Instrumentation \& Measurement, Sensor Network and Automation (IMSNA), 2012, pp. 393-397, doi: 10.1109/MSNA.2012.6324603.

[13] S. H. Ibrahim, "Hardware Implementation of 32-Bit High-Speed Direct Digital Frequency Synthesizer," The Scientific World Journal vol. 2014, 2014, doi: 10.1155/2014/131568.

[14] M. Rungruanganukul and T. Siriborvornratanakul, "Deep Learning Based Gesture Classification for Hand Physical Therapy Interactive Program," In: Duffy V. (eds) Digital Human Modeling and Applications in Health, Safety, Ergonomics and Risk Management. Posture, Motion and Health. HCII 2020. Lecture Notes in Computer Science, vol 12198. Springer, Cham, 2020, pp. 349-358, doi: 10.1007/978-3-030-49904-4_26.

[15] W. Haaswijk et al., "Deep Learning for Logic Optimization Algorithms," 2018 IEEE International Symposium on Circuits and Systems (ISCAS), 2018, pp. 1-4, doi: 10.1109/ISCAS.2018.8351885.

[16] Z. Wang, X. Luoand, and Z. Gong "Application of Deep Learning in Analog Circuit Sizing " in CSAI '18: Proceedings of the 2018 2nd International Conference on Computer cience and Artificial, Shenzhen, China, 2018, pp. 571-575, doi: $10.1145 / 3297156.3297160$.

[17] R. Palaniappan and P.T. Vanathi, "High performance parallel prefix adder for wider word lengths," Global Journal of Pure and Applied Mathematics, vol. 11, no. 2, pp. 733-743, 2015

[18] Y. Ma, S. Roy, J. Miao, J. Chen, and B. Yu, "Cross-Layer Optimization for High Speed Adders: A Pareto Driven Machine Learning Approach," in IEEE Transactions on Computer-Aided Design of Integrated Circuits and Systems, vol. 38, no. 12, Dec. pp. 2298-2311, 2019, doi: 10.1109/TCAD.2018.2878129.

[19] A. Surya, "Power and Delay Estimation of universal and Exclusive gates using Static and Dynamic CMOS Design," International Journal of Engineering and Advanced Technology, vol. 8, no. 6, pp. 2438-2448, 2019, doi: 10.35940/ijeat.F8538.088619.

[20] K. Ravali, N. R. Vijay, S. Jaggavarapu, and R. Sakthivel, "Low power XOR gate design and its applications," 2017 Fourth International Conference on Signal Processing, Communication and Networking (ICSCN), 2017, pp. 1-4, doi: 10.1109/ICSCN.2017.8085699.

[21] V. r. Ganjanaboyina, M. r. CH, V. S. M. Ganugaphati, and A. Y. Babu, "Design Of 3 Bit Adder Using 6 Transistors In Mentor Graphics," 2019 IEEE International Conference on Electrical, Computer and Communication Technologies (ICECCT), 2019, pp. 1-4, doi: 10.1109/ICECCT.2019.8869223.

[22] V. Dokania, R. Verma, M. Guduri, and A. Islam, "Design of 10T full adder cell for ultralow-power applications," Ain Shams Engineering Journal, vol. 9, no. 4, pp. 2363-2372, Dec. 2018, doi: 10.1016/j.asej.2017.05.004.

[23] A. H. Majeed, M. S. B. Zainal, E. Alkaldy, and D. Md. Nor, "Full Adder Circuit Design with Novel Lower Complexity XOR Gate in QCA Technology," Transactions on Electrical and Electronic Materials, vol. 21, pp. 198-207, 2020, doi: 10.1007/s42341-01900166-y.

[24] G. Manikannan, K. Mahendran, and P. Prabakaran, "Low Power High Speed Full Adder Cell with XOR/XNOR Logic Gates in 90nm Technology," International Conference on Technical Advancements in Computers (ICTACC), 2017, pp. 61-65, doi: 10.1109/ICTACC.2017.25.

[25] B. Mukherjee, B. Roy, A. Biswas, and A. Ghosal, "Design of a low power $4 \times 4$ multiplier based on five transistor (5-T) half adder, eight transistor (8-T) full adder \& two transistor (2-T) AND gate," Proceedings of the 2015 Third International Conference on Computer, Communication, Control and Information Technology (C3IT), 2015, pp. 1-5, doi: 10.1109/C3IT.2015.7060143.

[26] S. Malipatil, V. Maheshwari, and M. B. Chandra, "Area Optimization of CMOS Full Adder Design Using 3T XOR," 2020 International Conference on Wireless Communications Signal Processing and Networking (WiSPNET), 2020, pp. 192-194, doi: 10.1109/WiSPNET48689.2020.9198627.

[27] P. Chakali, A. Silivero, and N. Koppala, "Design of High Speed Six Transistor Full Adder using a Novel Two Transistor XOR Gates," International Journal of Advanced Research in Computer Science and Electronics Engineering, vol. 1, no. 5, pp. 104-108, 2012

[28] Sruthi, K. M. Akhila Raj, A. R. Madhulika, and A. S. Ajin, "Design of a 3-T Half Adder," International Journal of Electronics and Communication Engineering, vol. 3, no. 6, pp. 5-8, 2016, doi: 10.14445/23488549/IJECE-V3I6P103.

[29] V. R. Tirumalasetty and M. R. Machupalli, "Design and Analysis of Low Power High Speed 1-Bit Full Adder Cells for VLSI Applications," International Journal of Electronics, vol. 106, no. 4, pp 521-536, 2019, doi: 10.1080/00207217.2018.1545256.

[30] V. R. Trumalasetty and A. Srinivasulu, "16-BIT RCA Implementation Using Current Sink Restorer Structure," International Journal of Design, Analysis And Tools For Intergrated Circuits And Systems, vol. 4, no. 1, pp. 9-14, 2013.

[31] S. Ameta, V. Maurya, A. Hussain, and N. Agrawal, "Design and Analysis of 8-bit Carry Look Ahead Adder using TG and GDI Technology," International Journal of Innovative Research in Science, Engineering and Technology, vol. 6, no. 7, pp. 13848-13857, 2017. 
[32] G. Nayan, "A Comparative Analysis of 8-bit Novel Adder Architecture Design using Traditional CMOS and m-GDI technique," 2019 International Conference on Communication and Electronics Systems (ICCES), 2019, pp. 128-135, doi: 10.1109/ICCES45898.2019.9002573.

[33] A. T. U. Bhatt, N. Sharma, and S. Gite, "Comparative Study of Implementation of 8-bit Carry Select Adder using different Technologies," International Research Journal of Engineering and Technology, vol. 6, no. 12, pp. 243-247, 2019, [Online]. Available at: https://irjet.com/archives/V6/i12/IRJET-V6I1228.pdf.

\section{BIOGRAPHIES OF AUTHORS}

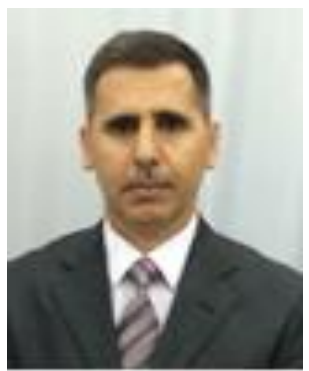

Salah Hasan Alkurwy (D) 8D SC P received his BSc. Degree in electrical engineering, MSc degree in electrical and electronic engineering from University of Belgrade/Serbia. He received his Ph.D. degree in Electrical, Electronic and System Engineering in 2015, from National University of Malaysia (UKM), Malaysia. Presently, he is an Associated professor and a head of the Department of Electronic Engineering, College of Engineering, University of Diyala/Iraq. His research Interest includes Digital circuits and system design, Microelectronics, digital electronics and HDL programming, Frequency Synthesis (DDFS). many scientific research articles published in international journals and conferences. He can be contacted at email: salahalkurwy@uodiyala.edu.iq

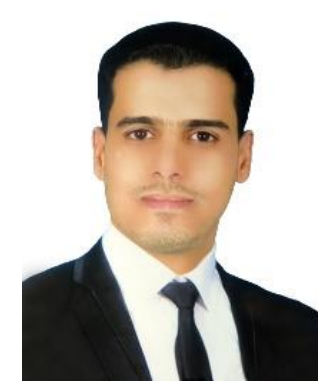

Isam Salah Hameed (i) S SC P received his BSc. Degree in electronic engineering from the University of Diyala, Iraq, in 2005. He received the MSc. Degree in electrical engineering at the University of Bridgeport, CT, USA, 2013. Currently, he is a lecturer in the department of the Electronic Engineering\College of Engineering $\backslash$ University of Diyala and his position is the rapporteur of the same department. His research interest is image processing, signal processing, DSP, digital VLSI, and PLC. He can be contacted at email: isamsh@uodiyala.edu.iq 